\title{
FERMIONIC INTEGRALS AND ANALYTIC SOLUTIONS FOR TWO-DIMENSIONAL ISING MODELS
}

\author{
V. N. Plechko \\ Joint Institute for Nuclear Research, JINR-Dubna, Bogoliubov Laboratory of Theoretical Physics \\ 141980, Dubna, Moscow Region, Russia
}

(Received January 28, 1997)

\begin{abstract}
We review some aspects of the fermionic interpretation of the two-dimensional Ising model. The use is made of the notion of the integral over the anticommuting Grassmann variables. For simple and more complicated 2D Ising lattices, the partition function can be expressed as a fermionic Gaussian integral. Equivalently, the 2D Ising model can be reformulated as a free-fermion theory on a lattice. For regular lattices the analytic solution then readily follows by passing to the momentum space for fermions. We also comment on the effective field-theoretical (continuum-limit) fermionic formulations for the 2D Ising models near the critical point.
\end{abstract}

Key words: Ising model, anticommuting Grassmann variables, critical point.

PACS number(s): 64.60.Cn, 75.10.-b, 05.30.Fk

\section{INTRODUCTION}

The two-dimensional (2D) Ising model in a zero magnetic field was first solved by Onsager in his celebrated paper [1]. This remarkable solution has already contributed much to our understanding of the nature of the second order phase transitions in magnets [2-6]. The original algebraic method used by Onsager, however, was sufficiently complicated. The modern approaches to the 2D Ising model are based merely on the fermionic interpretation of this model [7-23]. The fermionic structures in the 2D Ising model were first recognized within the transfer-matrix and combinatorial considerations $[7,8]$. It was realized later on that the notion of the integral over anticommuting Grassmann variables due to Berezin $[9,10]$ is a powerful tool to analyze the $2 \mathrm{D}$ Ising models [10-23]. Berezin himself was the first to apply the anticommuting variables to evaluate the partition function of the 2D Ising model on a rectangular lattice [10]. His method was based on the combinatorial considerations [10]. The fermionic approaches to the 2D Ising model have been developed later on by many authors [11-23]. In the forthcoming discussion we will merely follow a simple fermionic interpretation of the 2D Ising model introduced in [17-19]. The approach is based on the integration over the anticommuting Grassmann variables and the mirror-ordered factorization principle for the 2DIM density matrix. The method does not involve the traditional transfer-matrix or combinatorial considerations. Schematically, we have:

$$
\begin{aligned}
Q & =\operatorname{Sp}_{(\sigma)} Q(\sigma) \rightarrow \underset{(\sigma \mid a)}{\operatorname{Sp}_{(}} Q(\sigma \mid a) \\
& \rightarrow \underset{(a)}{\operatorname{Sp}} Q(a)=Q
\end{aligned}
$$

Here we start with the original spin partition function, $Q$, and introduce, in a special way, a set of new anticommuting Grassmann variables $(a)$ thus passing to a mixed $(\sigma \mid a)$ representation for $Q$. Eliminating the Ising spin variables in this mixed $(\sigma \mid a)$ representation, we obtain a purely fermionic expression for the same partition function $Q$. The partition function appears, in final form, as a Gaussian fermionic integral. Equivalently, the 2D Ising model is represented as a free-fermion field theory on a lattice [17-19]. For the homogeneous (translationally invariant) lattices the analytic solution for the partition function and free energy then readily follows by passing to the momentum space for fermions by means of Fourier substitution. In particular, this gives a few line derivation of the Onsager result [17]. The exact solution for a finite lattice on a torus (periodic boundary conditions in both directions) also follows within suitable modification of the fermionization procedure [18]. The decomposition of the partition function on a torus into a characteristic sum of four fermionic integrals follows here from a general rule of transposition of two Grassmann variable functions derived in Appendix A of [18]. The Ising models settled on the lattices with complicated local structures have been analyzed by the factorization method within the spinpolynomial interpretation of the problem [19]. We will pay more attention to this last case in sections $3-7$. For the application of the results of fermionic analysis [19] in studies of regularly diluted Ising ferromagnets also see $[25,26]$. It is also important that the method works in fact for the most general inhomogeneous distribution of the coupling parameters over the lattice bonds [17]. This may be of interest in studies of the disordered Ising models [20-23]. The Gaussian fermionic representations has been recently constructed as well for the inhomogeneous two-dimensional dimer problems [24].

In the straightforward variant of the method [17] we introduce Grassmann variables already at the first stages in order to decouple the local bond Boltzmann weights into separable factors called Grassmann factors (GFs). We then combine GFs with the same spin variables into separable groups all over the lattice and then sum over spin states in each group independently, thus passing to a purely fermionic representation for $Q$. In general, the factorization idea we apply resembles the idea of insertion of the Dirac unity, $\Sigma|a\rangle\langle a|=1$, in quantum mechanics. 
However, the situation in our case is more complicated since we deal here with Grassmann factors which are neither commuting nor anticommuting, in general, with each other. The key point of all the construction is then the mirror-ordering procedure for the global products of GFs [17]. This ordering procedure enables us to keep nearby few relevant factors with the same spin variable in the process of fermionization, which is the necessary condition so that we can actually eliminate spin degrees of freedom. The ordering procedure for global product of GFs is the most important part of the solution [17-19]. It might be worthwhile also mentioning that the lacking of a suitable ordering procedure for GFs in the 3D case is a serious obstacle to deal with the $3 \mathrm{D}$ Ising model within the fermionic factorization method. The same holds true for the 2D Ising model in a non-zero magnetic field.

If we start with factorization of the local bond Boltzmann weights, as we do for the standard rectangular lattice in $[17,18]$, we obtain the fermionic representation for $Q$ with four variables per site. Starting by the factorization of the bond weights, the number of fermionic variables per site will be even larger for more complicated lattices. An important modification of the method was introduced in [19], where we start with the factorization of the local cell weights presented by three-spin polynomials. The spin-polynomial interpretation for the $2 \mathrm{D}$ Ising models arises if we multiply few local weights forming elementary cell in $Q$. Effectively, this reduces the number of fluctuating degrees of freedom already on the level of the spin-variable formulation of the problem, prior to fermionization. Respectively, the Gaussian integral for $Q$ appears here with only two fermionic variables per site, see (4.1) below, which provides essential simplifications in the forthcoming analysis [19]. For a set of $2 \mathrm{D}$ Ising models, including rectangular, triangular and hexagonal Ising lattices as the simplest cases, this gives analytic solution in terms of the parameters of the three-spin polynomial characterizing elementary cell. The exact lattice integral (4.1) is also a suitable starting point to pass to the effective continuum-limit formulation near $T_{c}$. The continuum-limit formulation corresponds to the low-momentum sector of the exact lattice theory responsible for the critical-point singularities and the large-distance behaviour of correlations near $T_{c}$. The resulting theory is the massive 2D Majorana theory, with mass vanishing at $T_{c}$. By doubling of fermions in Majorana representation, we can pass as well to the 2D Dirac field theory of charged fermions. Below we comment on some aspects of the fermionic interpretation of the $2 \mathrm{D}$ Ising model in more detail. The fragments of the present discussion have been reported also in $[27,28]$. In the next section we outline shortly the basic rules of fermionic integration.

\section{GRASSMANN VARIABLES}

We remember that Grassmann variables are the purely anticommuting fermionic symbols. Given a set of Grassmann variables $a_{1}, a_{2}, \ldots, a_{N}$, we have $a_{i} a_{j}+a_{j} a_{i}=0$, $a_{j}^{2}=0$. The Beresin's rules of integration for one variable are [9]:

$$
\int d a_{j} \cdot a_{j}=1, \quad \int d a_{j} \cdot 1=0
$$

In the multidimensional integral, the differentials $d a_{1}, d a_{2}, \ldots, d a_{N}$ are again anticommuting with each other and with the variables. The basic relations of the Grassmannian analysis concern the Gaussian fermionic integrals $[9,10]$. The Gaussian integral of the first kind is related to the determinant:

$$
\int \prod_{j=1}^{N} d a_{j}^{*} d a_{j} \exp \left(\sum_{i=1}^{N} \sum_{j=1}^{N} a_{i} A_{i j} a_{j}^{*}\right)=\operatorname{det} \hat{A}
$$

where $\left\{a_{j}, a_{j}^{*}\right\}$ is a set of completely anticommuting Grassmann variables, the matrix in the exponential is arbitrary. The fermionic exponential here is assumed in the sense of its series expansion. The series terminates at some stage due to the property $a_{j}^{2}=0$. The correspondent finite polynomial for the exponential in (2.2) can be obtained also by multiplying the elementary factors $\exp \left(a_{i} A_{i j} a_{j}^{*}\right)=1+a_{i} A_{i j} a_{j}^{*}$. The appearance of the determinant in $(3)$ is due to the known interrelations between fermionic algebra and determinant combinatorics. By convention, the variables $a_{j}$ and $a_{j}^{*}$ can be considered as complex conjugated fermionic fields, otherwise these are simply independent variables. The Gaussian integral of the second kind, for real fermionic fields, is related to the Pfaffian of the associated skew-symmetric matrix:

$$
\begin{aligned}
& \int d a_{N} \ldots d a_{2} d a_{1} \exp \left(\frac{1}{2} \sum_{i=1}^{N} \sum_{j=1}^{N} a_{i} A_{i j} a_{j}\right)=\operatorname{Pfaff} \hat{A} \\
& A_{i j}=-A_{j i}
\end{aligned}
$$

The Pfaffian is some combinatorial polynomial in elements $A_{i j}$ which has been known in mathematics for a long time. Under another name, the Pfaffian is also well known in physics since the Pfaffian combinatorics is identical with that of the fermionic version of Wick's theorem. For any skew-symmetric matrix $\left(A_{i j}=-A_{j i}\right)$ we have:

$$
\operatorname{det} \hat{A}=(\operatorname{Pfaff} \hat{A})^{2} .
$$

This identity can be most easily proved just in terms of the fermionic integrals like (2.2) and (2.3). The fermionic averages (Green's functions) correspondent to the integrals (2.2) and (2.3) can be defined in a natural way. The linear superpositions of Grassmann variables are again Grassmann variables and it is possible to make a linear change of variables in the fermionic integrals. As compared with the rules of commuting analysis, the only difference is that Jacobian will now appear in the in- 


\section{N. PLECHKO}

verse power $[9,10]$. [It might be also interesting to compare (2.2) with the analogous Gaussian integral over the commuting complex-variable fields in common analysis]. In physical applications, the new fermionic variables of integration are often introduced by Fourier substitution (transformation to the momentum space).

\section{THE SPIN-POLYNOMIAL INTERPRETATION}

In order to construct the fermionic representation for $Q$ with the minimal number of fermionic variables per site, we start with the spin-polynomial formulation of the problem [19]. This can be most simply illustrated by an example of the Ising model on a triangular lattice. The triangular lattice can be viewed as a rectangular one with an additional diagonal interaction introduced in each rectangular cell. Let the lattice sites on the corresponding rectangular net be marked by integer Cartesian coordinates $m n$, with $m=1,2, \ldots, L, n=1,2, \ldots, L$ running in horizontal and vertical directions, respectively. Here $L$ is the lattice length, $N=L^{2}$ is the total number of sites and spins in a lattice. At final stages we assume $L^{2} \rightarrow \infty$. With each lattice site we associate the Ising spin variable $\sigma_{m n}= \pm 1$. The Hamiltonian of the Ising model on a triangular lattice is then given as follows:

$$
-\beta H(\sigma)=\sum_{m n}\left[b_{1} \sigma_{m n} \sigma_{m+1 n}+b_{2} \sigma_{m+1 n} \sigma_{m+1 n+1}+b_{3} \sigma_{m n} \sigma_{m+1 n+1}\right], \quad \beta=1 / k T,
$$

where $b_{\alpha}=J_{\alpha} / k T$ are the dimensionless coupling constants, $J_{\alpha}$ are the magnetic exchange energies, and $k T$ is the temperature in energy units. To be definite, in what follows we will merely keep in mind the ferromagnetic case, $b_{\alpha}>0$, though this restriction is not essential until we pass to the continuum limit formulations. The partition function and the free energy per site are:

$$
Z=\sum_{(\sigma)} \mathrm{e}^{-\beta H(\sigma)}, \quad-\beta f_{Z}=\lim _{N \rightarrow \infty} \frac{1}{N} \ln Z
$$

where the sum is taken over the $2^{N}$ spin configurations provided by $\sigma_{m n}= \pm 1$ at each site.

Noting the identity for the typical bond weight: $\mathrm{e}^{b \sigma \sigma^{\prime}}=\cosh b+\sinh b \cdot \sigma \sigma^{\prime}$, which readily follows from $\left(\sigma \sigma^{\prime}\right)^{2}=+1$, we find:

$$
Z=\left(2 \cosh b_{1} \cosh b_{2} \cosh b_{3}\right)^{N} Q, \quad N=L^{2} \rightarrow \infty
$$

where $Q$ is the reduced partition function:

$$
Q=\operatorname{Sp}_{(\sigma)}\left\{\prod_{m n}\left(1+t_{1} \sigma_{m n} \sigma_{m+1 n}\right)\left(1+t_{2} \sigma_{m+1 n} \sigma_{m+1 n+1}\right)\left(1+t_{3} \sigma_{m n} \sigma_{m+1 n+1}\right)\right\}
$$

where $t_{\alpha}=\tanh b_{\alpha}, b_{\alpha}=J_{\alpha} / k T$, and $S p_{(\sigma)}$ is the properly normalized spin averaging [19]:

$$
\underset{(\sigma)}{\operatorname{Sp}}(\ldots)=\prod_{m n} \operatorname{Sp}_{\left(\sigma_{m n}\right)}(\ldots)=2^{-N} \sum_{(\sigma)}(\ldots), \quad \underset{\left(\sigma_{m n}\right)}{\operatorname{Sp}_{0}}(\ldots)=\frac{1}{2} \sum_{\sigma_{m n}= \pm 1}(\ldots)
$$

the local averaging is normalized here in such a way that $\operatorname{Sp}(1)=1$, also note that $\operatorname{Sp}\left(\sigma_{m n}\right)=0$. The reduced partition function $Q$ will be the main subject of our interest.

In order to pass to the spin-polynomial interpretation, we have to multiply the three bond weights forming a triangular cell in $Q$. Let us introduce the local enumeration of sites at our rectangular net:

$$
\left(\sigma_{1}\left|\sigma_{2}\right| \sigma_{3} \mid \sigma_{4}\right) \leftrightarrow\left(\sigma_{m n}\left|\sigma_{m+1 n}\right| \sigma_{m+1 n+1} \mid \sigma_{m n+1}\right)
$$

The elementary cell in (3.1) and (3.4) is formed by a triangle of spins $\left(\sigma_{1}, \sigma_{2}, \sigma_{3}\right)_{m n}$. Noting that $\sigma_{j}= \pm 1$, and hence $\sigma_{j}^{2}=1$, we can multiply the weights in (3.4) making use of the properties like $\sigma_{1} \sigma_{2} \cdot \sigma_{2} \sigma_{3}=\sigma_{1} \sigma_{3}$, etc. For the product of the three bond weights from (3.4) we then obtain the three-spin polynomial as is given in (3.7) below. This polynomial can be considered as the effective Boltzmann weight of a triangular elementary cell taken as a whole: 


$$
\begin{aligned}
P_{123}(\sigma) & =\left(1+t_{1} \sigma_{1} \sigma_{2}\right)\left(1+t_{2} \sigma_{2} \sigma_{3}\right)\left(1+t_{3} \sigma_{1} \sigma_{3}\right) \\
& =\left(1+t_{1} t_{2} t_{3}\right)+\left(t_{1}+t_{2} t_{3}\right) \sigma_{1} \sigma_{2}+\left(t_{2}+t_{1} t_{3}\right) \sigma_{2} \sigma_{3}+\left(t_{3}+t_{1} t_{2}\right) \sigma_{1} \sigma_{3} \\
& =\alpha_{0}+\alpha_{1} \sigma_{1} \sigma_{2}+\alpha_{2} \sigma_{2} \sigma_{3}+\alpha_{3} \sigma_{1} \sigma_{3} .
\end{aligned}
$$

In the last line we write the three-spin polynomial in general notation, for the given case of a triangular lattice we have:

$$
\alpha_{0}=1+t_{1} t_{2} t_{3}, \quad \alpha_{1}=t_{1}+t_{2} t_{3}, \quad \alpha_{2}=t_{2}+t_{1} t_{3}, \quad \alpha_{3}=t_{3}+t_{1} t_{2},
$$

while the case of a rectangular lattice follows with $t_{3}=0$, which corresponds to $b_{3}=0$ in the Hamiltonian (3.1). For a rectangular lattice we have: $\alpha_{0}=1, \alpha_{1}=t_{1}, \alpha_{2}=t_{2}, \alpha_{3}=t_{1} t_{2}$. The hexagonal and more complicated lattices can be characterized by the three-spin polynomials like (3.7) as well, with their own sets of the $\alpha$-parameters. Thus we come to the three-spin polynomial partition function [19]:

$$
Q=\operatorname{Sp}_{(\sigma)} \prod_{m n}\left(\alpha_{0}+\alpha_{1} \sigma_{m n} \sigma_{m+1 n}+\alpha_{2} \sigma_{m+1 n} \sigma_{m+1 n+1}+\alpha_{3} \sigma_{m n} \sigma_{m+1 n+1}\right) .
$$

In what follows we assume $\alpha_{0}, \alpha_{1}, \alpha_{2}, \alpha_{3}$ to be arbitrary parameters. Particular Ising lattices are specified by the choice of the $\alpha$-parameters $[19,25,26]$.

\section{FREE-FERMION REPRESENTATION AND ANALYTIC RESULTS (THE LATTICE CASE)}

The three-spin polynomial partition function (3.9) can be transformed into a fermionic Gaussian integral following (1.1). The starting point is factorization of the local polynomial weights in (3.9). We need only two variables per site in the process of fermionization. The partition function then appears as the following Gaussian fermionic integral [19]:

$$
\begin{aligned}
Q & =\int \prod_{m n} d c_{m n}^{*} d c_{m n} \exp \sum_{m n}\left[\left(\alpha_{0} c_{m n} c_{m n}^{*}-\alpha_{1} c_{m n} c_{m-1 n}^{*}-\alpha_{2} c_{m n} c_{m n-1}^{*}\right.\right. \\
& \left.\left.-\alpha_{3} c_{m n} c_{m-1 n-1}^{*}\right)-\alpha_{1} c_{m n} c_{m-1 n}-\alpha_{2} c_{m n}^{*} c_{m n-1}^{*}\right]
\end{aligned}
$$

where $c_{m n}, c_{m n}^{*}$ are the totally anticommuting Grassmann variables, two per site. The free-fermion representation (4.1) is exact. Integral (4.1) is equivalent to the original partition function (3.9) up to the boundary effects, which are inessential as $L^{2} \rightarrow \infty$. The explicit evaluation of integral (4.1) can be performed by passing to the momentum space by means of Fourier substitution:

$$
c_{m n}=\frac{1}{L} \sum_{p q} c_{p q} \mathrm{e}^{i \frac{2 \pi}{L}(m p+n q)}, \quad c_{m n}^{*}=\frac{1}{L} \sum_{p q} c_{p q}^{*} \mathrm{e}^{-i \frac{2 \pi}{L}(m p+n q)} .
$$

Here $c_{p q}, c_{p q}^{*}$ are the new fermionic variables of integration. Integral (4.1) now appears in the form of

$$
\begin{aligned}
Q & =\int \prod_{p q} d c_{p q}^{*} d c_{p q} \exp \sum_{p q}\left[c_{p q} c_{p q}^{*}\left(\alpha_{0}-\alpha_{1} \mathrm{e}^{i \frac{2 \pi p}{L}}-\alpha_{2} \mathrm{e}^{i \frac{2 \pi q}{L}}-\alpha_{3} \mathrm{e}^{i \frac{2 \pi}{L}(p+q)}\right)\right. \\
& \left.-\alpha_{1} c_{p q} c_{L-p L-q} \mathrm{e}^{i \frac{2 \pi p}{L}}-\alpha_{2} c_{L-p L-q}^{*} c_{p q}^{*} \mathrm{e}^{i \frac{2 \pi q}{L}}\right] .
\end{aligned}
$$

Note that the fermionic measure transforms in a trivial way by passing from (4.1) to (4.3). This is because the Jacobian of substitution (4.2) is unity which follows from the orthogonality of the Fourier eigenfunctions.

It is easy to see that the integral (4.3) decouples into a product of simple low-dimensional integrals. Since the variables with the momenta $p q$ and $L-p L-q$ interact in (4.3), we have to single out in the fermionic action in (4.3) the combined $(p q \mid L-p L-q)$ term. Let $S_{p q}$ be the term already given explicitly in the $p q$ sum above. It then follows that the integral (4.3) is the product of the following independent factors: 


\section{N. PLECHKO}

$$
Q_{p q}^{2}=\int D_{p q} \exp \left(S_{p q}+S_{L-p L-q}\right), \quad D_{p q}=d c_{L-p L-q}^{*} d c_{L-p L-q} d c_{p q}^{*} d c_{p q}
$$

The elementary integral (4.4) can be readily evaluated by elementary tools (for instance, simply using definitions (2.1)) and its value is given as a factor in the product (4.5) below. By comparing the fermionic measures in (4.3) and (4.4), it follows that the partition function itself, $Q$, arises if we multiply the factors (4.4) over only one half of the points in the momentum space. That is, we have to multiply the factors (4.4) in such a way that if the given mode $p q$ is already included into the product, then the conjugated mode $L-p L-q$ is not to be included, and vice versa. Respectively, the total product of factors (4.4) over the complete set of momentum modes, $0 \leq p, q \leq L-1$, will yield the squared partition function. Thus, for the partition function (4.1) we find [19]:

$$
\begin{aligned}
Q^{2} & =\prod_{p=0}^{L-1} \prod_{q=0}^{L-1}\left[\left(\alpha_{0}^{2}+\alpha_{1}^{2}+\alpha_{2}^{2}+\alpha_{3}^{2}\right)-2\left(\alpha_{0} \alpha_{1}-\alpha_{2} \alpha_{3}\right) \cos \frac{2 \pi p}{L}\right. \\
& \left.-2\left(\alpha_{0} \alpha_{2}-\alpha_{1} \alpha_{3}\right) \cos \frac{2 \pi q}{L}-2\left(\alpha_{0} \alpha_{3}-\alpha_{1} \alpha_{2}\right) \cos \frac{2 \pi(p+q)}{L}\right] .
\end{aligned}
$$

Respectively, the free energy per site is:

$$
\begin{aligned}
-\beta f_{Q} & =\lim _{L \rightarrow \infty}\left(\frac{1}{L^{2}} \ln Q\right) \\
& =\frac{1}{2} \int_{0}^{2 \pi} \int_{0}^{2 \pi} \frac{d p}{2 \pi} \frac{d q}{2 \pi} \ln \left[\left(\alpha_{0}^{2}+\alpha_{1}^{2}+\alpha_{2}^{2}+\alpha_{3}^{2}\right)-2\left(\alpha_{0} \alpha_{1}-\alpha_{2} \alpha_{3}\right) \cos p\right. \\
& \left.-2\left(\alpha_{0} \alpha_{2}-\alpha_{1} \alpha_{3}\right) \cos q-2\left(\alpha_{0} \alpha_{3}-\alpha_{1} \alpha_{2}\right) \cos (p+q)\right] .
\end{aligned}
$$

In particular, the exact solutions for the rectangular, triangular, and hexagonal Ising lattices follow from (4.6) under the correspondent specifications of the parameters $\alpha_{j}[19]$.

Here we consider as an illustration the simplest case of the standard rectangular lattice. For this lattice, we have $\alpha_{0}, \alpha_{1}, \alpha_{2}, \alpha_{3} \leftrightarrow 1, t_{1}, t_{2}, t_{1} t_{2}$. From (4.6), the free energy is:

$$
\begin{aligned}
-\beta f_{Q} \mid \text { rect } & =\frac{1}{2} \int_{0}^{2 \pi} \int_{0}^{2 \pi} \frac{d p}{2 \pi} \frac{d q}{2 \pi} \ln \left[\left(1+t_{1}^{2}\right)\left(1+t_{2}^{2}\right)-2 t_{1}\left(1-t_{2}^{2}\right) \cos p\right. \\
& \left.-2 t_{2}\left(1-t_{1}^{2}\right) \cos q\right] .
\end{aligned}
$$

The free energy (4.7) is associated with the reduced partition function, $Q$, while the true partition function is $Z=\left(2 c_{1} c_{2}\right)^{N} Q$, see (3.3) and (3.4) with $t_{3}=0$. Also we remember the identities like $c_{\alpha}^{2}\left(1+t_{\alpha}^{2}\right)=\cosh 2 b_{\alpha}$, $2 t_{\alpha} c_{\alpha}^{2}=\sinh 2 b_{\alpha}$, etc, with $b_{\alpha}=J_{\alpha} / k T$. From (4.7), the true free energy per site then appears in the form of

$$
\begin{aligned}
-\left.\beta f_{Z}\right|_{\text {rect }} & =\ln 2+\frac{1}{2} \int_{0}^{2 \pi} \int_{0}^{2 \pi} \frac{d p}{2 \pi} \frac{d q}{2 \pi} \ln \left[\cosh 2 b_{1} \cos 2 b_{2}-\sinh 2 b_{1} \cos p\right. \\
& \left.-\sinh 2 b_{2} \cos q\right] .
\end{aligned}
$$

This is the famous Onsager solution for the free energy of the $2 \mathrm{D}$ Ising model on a rectangular lattice [1]. An interesting comment on the structure of this solution follows immediately after equation (108) in [1].

As follows from (4.7) for a rectangular lattice the critical point is fixed by the condition:

$$
1-t_{1}-t_{2}-t_{1} t_{2}=0, \quad t_{\alpha}=\tanh \left(J_{\alpha} / k T\right)
$$

equivalently, this condition can be written in the form of

$$
\sinh \left(2 J_{1} / k T\right) \sinh \left(2 J_{2} / k T\right)=1
$$

which rather corresponds to the solution in the form of (4.8). In both formulations the ferromagnetic case is assumed, $b_{1}, b_{2}>0$, equivalently, $t_{1}, t_{2}>0$. The free 
energy is known, the specific heat can be obtained by differentiation with respect to the temperature: $C / k=$ $\beta^{2}\left(\partial^{2}(-\beta f) / \partial \beta^{2}\right)$, where $C / k$ is the dimensionless specific heat, $\beta=1 / k T$, and $k$ is the Boltzmann constant. The singularity in the specific heat appears to be logarithmic near $T_{c}: C / k \sim|\ln | \tau||, \tau=\left(T-T_{c}\right) / T_{c} \rightarrow 0$, as it can be deduced from (4.7) and/or (4.8). Also see the discussion in the next section.

\section{THE SYMMETRIES AND THE CRITICAL POINT}

The symmetries provided by the above exact solution (4.5) and (4.6), and the closely related question on the location of the critical point, have an interesting interpretation within the spin-polynomial language [19]. Making use of the local enumeration of sites in the elementary cell, see (3.6), the cell weight in the density matrix in (3.9) is given as the three-spin polynomial:

$$
P_{123}(\sigma)=\alpha_{0}+\alpha_{1} \sigma_{1} \sigma_{2}+\alpha_{2} \sigma_{2} \sigma_{3}+\alpha_{3} \sigma_{1} \sigma_{3} .
$$

It appears also to be useful to introduce the associated three-spin polynomial:

$$
\begin{aligned}
& \alpha_{0}^{*}=\frac{1}{2}\left(\alpha_{0}+\alpha_{1}+\alpha_{2}+\alpha_{3}\right), \\
& \alpha_{1}^{*}=\frac{1}{2}\left(\alpha_{0}+\alpha_{1}-\alpha_{2}-\alpha_{3}\right), \\
& \alpha_{2}^{*}=\frac{1}{2}\left(\alpha_{0}-\alpha_{1}+\alpha_{2}-\alpha_{3}\right), \\
& \alpha_{3}^{*}=\frac{1}{2}\left(\alpha_{0}-\alpha_{1}-\alpha_{2}+\alpha_{3}\right),
\end{aligned}
$$

$\bar{\alpha}_{0}=\frac{1}{2}\left(\alpha_{0}-\alpha_{1}-\alpha_{2}-\alpha_{3}\right)$,
$\bar{\alpha}_{1}=\frac{1}{2}\left(\alpha_{0}-\alpha_{1}+\alpha_{2}+\alpha_{3}\right)$,
$\bar{\alpha}_{2}=\frac{1}{2}\left(\alpha_{0}+\alpha_{1}-\alpha_{2}+\alpha_{3}\right)$,
$\bar{\alpha}_{3}=\frac{1}{2}\left(\alpha_{0}+\alpha_{1}+\alpha_{2}-\alpha_{3}\right)$.

$$
F_{123}(\sigma)=\alpha_{0}-\alpha_{1} \sigma_{1} \sigma_{2}-\alpha_{2} \sigma_{2} \sigma_{3}-\alpha_{3} \sigma_{1} \sigma_{3},
$$

and we note the following interesting identity [19]:

$$
\begin{aligned}
& \left(F_{123}(\sigma)\right)^{2}=\left(\alpha_{0}-\alpha_{1} \sigma_{1} \sigma_{2}-\alpha_{2} \sigma_{2} \sigma_{3}-\alpha_{3} \sigma_{1} \sigma_{3}\right)^{2} \\
& =\left(\alpha_{0}^{2}+\alpha_{1}^{2}+\alpha_{2}^{2}+\alpha_{3}^{2}\right)-2\left(\alpha_{0} \alpha_{1}-\alpha_{2} \alpha_{3}\right) \sigma_{1} \sigma_{2} \\
& -2\left(\alpha_{0} \alpha_{2}-\alpha_{1} \alpha_{3}\right) \sigma_{2} \sigma_{3}-2\left(\alpha_{0} \alpha_{3}-\alpha_{1} \alpha_{2}\right) \sigma_{1} \sigma_{3} .
\end{aligned}
$$

It is seen that the combinations of the $\alpha$-parameters occurring in $\left(F_{123}\right)^{2}$ are exactly the same as in the momentum modes $Q^{2}(p \mid q)$ in (4.6):

$$
\begin{aligned}
& Q^{2}(p \mid q)=\left(\alpha_{0}^{2}+\alpha_{1}^{2}+\alpha_{2}^{2}+\alpha_{3}^{2}\right) \\
& -2\left(\alpha_{0} \alpha_{1}-\alpha_{2} \alpha_{3}\right) \cos p-2\left(\alpha_{0} \alpha_{2}-\alpha_{1} \alpha_{3}\right) \cos q \\
& -2\left(\alpha_{0} \alpha_{3}-\alpha_{1} \alpha_{2}\right) \cos (p+q),
\end{aligned}
$$

where $0 \leq p, q \leq 2 \pi$, the limit $L^{2} \rightarrow \infty$ is already assumed.

It appears that the following combinations of the $\alpha$ parameters play an important role in discussing the symmetries and the critical point [19]:
The parameters $\alpha^{*}$ and $\bar{\alpha}$ are in fact the eigenvalues of the polynomials $\frac{1}{2} P_{123}$ and $\frac{1}{2} F_{123}$, respectively. By the "eigenvalues" we mean the four numbers which takes the polynomial as the spin variables run over their permissible values \pm 1 . We note also the following important identity [19]:

$$
\bar{\alpha}_{0} \bar{\alpha}_{1} \bar{\alpha}_{2} \bar{\alpha}_{3}=\alpha_{0}^{*} \alpha_{1}^{*} \alpha_{2}^{*} \alpha_{3}^{*}-\alpha_{0} \alpha_{1} \alpha_{2} \alpha_{3} .
$$

There are some evident symmetries in the solution (4.6). For instance, the free energy (4.6) is a symmetric function with respect to arbitrary permutations of the $\alpha_{0}, \alpha_{1}, \alpha_{2}, \alpha_{3}$ parameters. We can as well change the signs of any two of them, with $-\beta f_{Q}$ unchanged. There is also a less evident hidden symmetry in the solution, corresponding to the Kramers-Wannier duality in the case of the standard rectangular lattice. Namely, the partition function $Q\{\alpha\}$ is invariant under the transformation $\alpha_{j} \leftrightarrow \alpha_{j}^{*}$. This symmetry in fact holds already for the parameters of the separable fermionic modes (5.4), 


\section{N. PLECHKO}

It can be shown also that if all bond interactions are ferromagnetic then $\bar{\alpha}_{1}, \bar{\alpha}_{2}, \bar{\alpha}_{3}$ never become zero, and the critical point can only be associated with $\bar{\alpha}_{0}=0$. Thus, for the purely ferromagnetic interactions the unique critical pont is definite by the equation:

$$
2 \bar{\alpha}_{0}=\alpha_{0}-\alpha_{1}-\alpha_{2}-\alpha_{3}=0 .
$$

The criticality conditions with $\bar{\alpha}_{k}=0, k=1,2,3$, can only be realized when the antiferromagnetic interactions are involved in the Hamiltonian.

If the critical point is associated with $\bar{\alpha}_{j}=0$, then the singular part of the free energy (4.6) near $T_{c}$ is given as follows [19]:

$$
-\left.\beta f_{Q}\right|_{\text {singular }}=\frac{\left(2 \bar{\alpha}_{j}\right)^{2}}{16 \pi \sqrt{\left(\alpha_{0} \alpha_{1} \alpha_{2} \alpha_{3}\right)_{c}}} \ln \frac{\text { const }}{\left(2 \bar{\alpha}_{\mathrm{j}}\right)^{2}}
$$

which implies that, near $T_{c}$, in the order of magnitude $-\beta f_{Q} \sim \tau^{2} \ln \tau^{2}$, where $\tau \sim\left|T-T_{c}\right|$. The specific heat has thus the log-type singularity, $C \sim|\ln \tau|$ as $T \rightarrow T_{c}$ (Onsager, 1944).

It is seen that the eigenvalues (5.5) play an important role, but it is not so clear, in fact, how the role of the polynomial $F_{123}$ can be understood in a less formal way, at the level of the original spin-variable formulation of the problem, prior to the analytic solution. The special role which the parameters (5.5) play in the inherent structure of the $2 \mathrm{D}$ Ising model is confirmed by the expression for the spontaneous magnetization. The 8th power of the spontaneous magnetization $M=\left\langle\sigma_{m n}\right\rangle$ for model (3.9) is given by the following very simple expression [19]:

$$
M^{8}=(-1) \frac{\bar{\alpha}_{0} \bar{\alpha}_{1} \bar{\alpha}_{2} \bar{\alpha}_{3}}{\alpha_{0} \alpha_{1} \alpha_{2} \alpha_{3}}=1-\frac{\alpha_{0}^{*} \alpha_{1}^{*} \alpha_{2}^{*} \alpha_{3}^{*}}{\alpha_{0} \alpha_{1} \alpha_{2} \alpha_{3}}
$$

This expression for $M^{8}$ holds true when the right hand side varies between 0 and 1 , and $M^{8}=0$ otherwise. It is easy to check that the known expressions for the spontaneous magnetizations of the rectangular, triangular, and hexagonal lattices follow easily from (5.10) as particular cases. From (5.10) we find $M \sim \tau^{\frac{1}{8}}$ as $\tau \sim\left|T-T_{c}\right| \rightarrow 0$, with the universal value of the critical index $\beta=1 / 8$ for the magnetization at the critical isobar. What are the hidden reasons for such a simple expression for $M^{8}$, this is yet unknown.

\section{MAJORANA FIELDS}

The fermionic integral for $Q$ given in (4.1) also appears to be a suitable starting point to formulate the continuum limit field theories for the correspondent models near $T_{c}$ [27]. For other approaches to the continuumlimit formulation of the 2D Ising model also see [15], [16], [22]. Assuming the purely ferromagnetic case, with $\alpha_{0}, \alpha_{1}, \alpha_{2}, \alpha_{3}$ all positive, we write once again the exact lattice action from (4.1) as follows:

$$
\begin{aligned}
S(c) & =\sum_{m n}\left[\left(\alpha_{0}-\alpha_{1}-\alpha_{2}-\alpha_{3}\right) c_{m n} c_{m n}^{*}+\alpha_{1} c_{m n}\left(c_{m n}^{*}-c_{m-1 n}^{*}\right)\right. \\
& +\alpha_{2} c_{m n}\left(c_{m n}^{*}-c_{m n-1}^{*}\right)+\alpha_{3} c_{m n}\left(c_{m n}^{*}-c_{m-1 n-1}^{*}\right) \\
& \left.+\alpha_{1} c_{m n}\left(c_{m n}-c_{m-1 n}\right)+\alpha_{2} c_{m n}^{*}\left(c_{m n}^{*}-c_{m n-1}^{*}\right)\right]
\end{aligned}
$$

with $c_{m n}^{2}=c_{m n}^{* 2}=0$. Let us define the lattice derivatives as follows: $\partial_{m} x_{m n}=x_{m n}-x_{m-1 n}, \partial_{n} x_{m n}=x_{m n}-x_{m n-1}$, also note that $x_{m n}-x_{m-1 n-1}=\partial_{m} x_{m n}+\partial_{n} x_{m n}-\partial_{m} \partial_{n} x_{m n}$. Introducing the new notation for the fermionic fields, $c_{m n}, c_{m n}^{*} \rightarrow \psi_{m n}, \bar{\psi}_{m n}$, we find action (6.1) in the form:

$$
\begin{gathered}
S(\psi)=\sum_{m n}\left[\underline{m} \psi_{m n} \bar{\psi}_{m n}+\lambda_{1} \psi_{m n} \partial_{m} \bar{\psi}_{m n}+\lambda_{2} \psi_{m n} \partial_{n} \bar{\psi}_{m n}\right. \\
\left.-\alpha_{3} \psi_{m n} \partial_{m} \partial_{n} \bar{\psi}_{m n}+\alpha_{1} \psi_{m n} \partial_{m} \psi_{m n}+\alpha_{2} \bar{\psi}_{m n} \partial_{n} \bar{\psi}_{m n}\right], \\
\lambda_{1}=\alpha_{1}+\alpha_{3}, \quad \lambda_{2}=\alpha_{2}+\alpha_{3}, \quad \underline{m}=\alpha_{0}-\alpha_{1}-\alpha_{2}-\alpha_{3}=2 \bar{\alpha}_{0} .
\end{gathered}
$$

It is easy to recognize in this still exact lattice action a typical relativistic field-theoretical like structure with the mass term and kinetic part. Since we assume the ferromagnetic case, the criticality condition is $2 \bar{\alpha}_{0}=0$, see $(5.8)$. The parameter $\underline{m}=\alpha_{0}-\alpha_{1}-\alpha_{2}-\alpha_{3}=2 \bar{\alpha}_{0}$ plays the role of mass in the field-theoretical interpretation. The mass vanishes at the critical point, $\underline{m} \sim\left|T-T_{c}\right| \rightarrow 0$ as $T \rightarrow T_{c}$. The ordered phase corresponds to $\underline{m}<0$.

Taking in (6.2) the formal limit to the continuum Euclidean space, with $(m n) \rightarrow x=\left(x_{1} \mid x_{2}\right), \psi_{m n} \rightarrow \psi(x)=$ $\psi\left(x_{1} \mid x_{2}\right)$, and $\partial_{m} \rightarrow \partial_{1}=\partial / \partial x_{1}, \partial_{n} \rightarrow \partial_{2}=\partial / \partial x_{2}$, and neglecting the second order kinetic term with $\partial_{1} \partial_{2}$, we obtain the Majorana like fermionic action of the correspondent continuum theory: 


$$
\begin{array}{r}
S=\int d^{2} x\left[\underline{m} \psi(x) \bar{\psi}(x)+\lambda_{1} \psi(x) \partial_{1} \bar{\psi}(x)+\lambda_{2} \psi(x) \partial_{2} \bar{\psi}(x)\right. \\
\left.+\alpha_{1} \psi(x) \partial_{1} \psi(x)+\alpha_{2} \bar{\psi}(x) \partial_{2} \bar{\psi}(x)\right]
\end{array}
$$

where the parameters $\lambda_{1}, \lambda_{2}, \underline{m}$ are defined in (6.2). A nonstandard feature in this action are the "nondiagonal" kinetic terms $\psi \partial_{1} \bar{\psi}, \psi \partial_{2} \bar{\psi}$. These terms can be eliminated by a suitable linear transformation of the fields: $\psi=$ $\gamma \psi_{1}+\eta \psi_{2}, \bar{\psi}=\bar{\gamma} \psi_{1}+\bar{\eta} \psi_{2}$, where $\psi_{1}, \psi_{2}$ are new Majorana components. The condition that the nondiagonal like terms will not appear in (6.4) is $\gamma \bar{\gamma}=\eta \bar{\eta}$. Omitting further details, after a suitable transformation of the fields and the axis in the $d^{2} x$ space, from (6.3) we obtain Majorana action in the canonical form (cf. $\left.[15,16]\right)$ :

$$
\begin{aligned}
& S=\int d^{2} x\left[\bar{m} \psi_{1}(x) \psi_{2}(x)+\psi_{1}(x) \partial_{0} \psi_{1}(x)-\psi_{2}(x) \bar{\partial}_{0} \psi_{2}(x)\right], \\
& \partial_{0}=\frac{1}{2}\left(\partial_{1}+i \partial_{2}\right), \quad \bar{\partial}_{0}=\frac{1}{2}\left(\partial_{1}-i \partial_{2}\right), \text { 2D Majorana },
\end{aligned}
$$

with the rescaled mass:

$$
\bar{m}=\frac{\alpha_{0}-\alpha_{1}-\alpha_{2}-\alpha_{3}}{\left(2 \sqrt{\left(\alpha_{0} \alpha_{1} \alpha_{2} \alpha_{3}\right)_{c}}\right)^{1 / 2}}
$$

The 2D Ising model is presented now as a field theory of free massive two-component Majorana fermions in Euclidean $d^{2} x$ space. The new Majorana fields in this representation, $\psi_{1}, \psi_{2}$, are the linearly transformed fields $\psi, \bar{\psi}$ from (6.3). The axis of the $d^{2} x$ space are also rescaled and rotated as we pass from (6.3) to (6.4). Respectively, the mass is rescaled according to (6.5), here ()$_{c}$ means the criticality condition $\left(\alpha_{0}-\alpha_{1}-\alpha_{2}-\alpha_{3}\right)_{c}=0$.

In matrix notation, the Majorana action (6.4) becomes:

$$
S_{\text {major }}=\frac{1}{2} \int d^{2} x\left(\begin{array}{l}
\psi_{1} \\
\psi_{2}
\end{array}\right)\left[\bar{m}\left(\begin{array}{rr}
0 & 1 \\
-1 & 0
\end{array}\right)+\left(\begin{array}{cc}
\partial_{1}+i \partial_{2} & 0 \\
0 & -\partial_{1}+i \partial_{2}
\end{array}\right)\right]\left(\begin{array}{l}
\psi_{1} \\
\psi_{2}
\end{array}\right) .
$$

Introducing the standard Pauli matrices:

$$
\sigma_{1}=\left(\begin{array}{ll}
0 & 1 \\
1 & 0
\end{array}\right), \quad \sigma_{2}=\left(\begin{array}{rr}
0 & -i \\
i & 0
\end{array}\right), \quad \sigma_{3}=\left(\begin{array}{rr}
1 & 0 \\
0 & -1
\end{array}\right)
$$

the action (6.6) can be written also in the form:

$$
S_{\text {major }}=\frac{1}{2} \int d^{2} x \Psi(x)\left[\bar{m}\left(i \sigma_{2}\right)+\partial_{1}\left(\sigma_{3}\right)+i \partial_{2}(1)\right] \Psi(x), \quad \Psi(x)=\left(\begin{array}{c}
\psi_{1} \\
\psi_{2}
\end{array}\right),
$$

or in the form:

$$
S_{\text {major }}=\frac{1}{2} \int d^{2} x \tilde{\Psi}(x)\left[\bar{m}+\partial_{1}\left(\sigma_{1}\right)+\partial_{2}\left(\sigma_{2}\right)\right] \Psi(x), \quad \tilde{\Psi}(x)=\Psi(x)\left(i \sigma_{2}\right) .
$$

Introducing the 2D gamma-matrices in a natural way: $\gamma_{1}=\sigma_{1}, \gamma_{2}=\sigma_{2}$, the action (6.9) becomes:

$$
S_{\text {major }}=\frac{1}{2} \int d^{2} x \tilde{\Psi}(x)[\bar{m}+\hat{\partial}] \Psi(x), \quad \hat{\partial}=\gamma_{1} \partial_{1}+\gamma_{2} \partial_{2}, \quad \hat{\partial}^{2}=\partial_{1}^{2}+\partial_{2}^{2},
$$

which is the 2D Majorana action written in the standard form assumed in relativistic field theory. Notice that the conjugated Majorana spinors $\tilde{\Psi}$ and $\Psi$ here are not the truly independent fields since they both are built from the same component fields $\psi_{1}, \psi_{2}$. The truly independent left and right spinors $\bar{\Psi}$ and $\Psi$ appear if pass to the Dirac theory. This is commented in the next section. 


\section{N. PLECHKO}

\section{THE DIRAC FIELDS}

We can pass to the Dirac field theory of charged fermions by doubling the number of fermions in the Majorana representation (6.4)-(6.10). On a formal level, this corresponds to passing from the Pfaffian like Gaussian integral to the determinantal Gaussian integral according to the identity $|\mathrm{Pfaff} A|^{2}=\operatorname{det} A$, see (2.4). To this end, we take the two identical copies $S^{\prime}$ and $S^{\prime \prime}$ of the Majorana action (6.10) and consider the combined action $S_{\text {dirac }}=S^{\prime}+S^{\prime \prime}$. In this combined action we introduce the new Dirac fermionic fields $\Psi=\left(\psi_{1} \mid \psi_{2}\right)$ and $\bar{\Psi}=\left(\psi_{1}^{*} \mid \psi_{2}^{*}\right)$ by substitution:

$$
\Psi=\frac{1}{\sqrt{2}}\left(\Psi^{\prime}+i \Psi^{\prime \prime}\right), \quad \bar{\Psi}=\frac{1}{\sqrt{2}}\left(\tilde{\Psi}^{\prime}-i \tilde{\Psi}^{\prime \prime}\right)
$$

where $\Psi^{\prime}, \Psi^{\prime \prime}, \tilde{\Psi}^{\prime}, \tilde{\Psi}^{\prime \prime}$ are the original Majorana spinors. In terms of the new fields the combined action $S_{\mathrm{dirac}}=$ $\left(S^{\prime}+S^{\prime \prime}\right)$ majorana becomes:

$$
S_{\text {dirac }}=\int d^{2} x \bar{\Psi}(x)[\bar{m}+\hat{\partial}] \Psi(x), \quad \hat{\partial}=\gamma_{1} \partial_{1}+\gamma_{2} \partial_{2}
$$

where the gamma matrices are the same as in (6.10). If written in components, the substitution inverse to (7.1) takes the form:

$$
\begin{aligned}
& \psi_{1}^{\prime}=\frac{1}{\sqrt{2}}\left(\psi_{1}+\psi_{2}^{*}\right), \quad i \psi_{1}^{\prime \prime}=\frac{1}{\sqrt{2}}\left(\psi_{1}-\psi_{2}^{*}\right), \\
& \psi_{2}^{\prime}=\frac{1}{\sqrt{2}}\left(\psi_{2}-\psi_{1}^{*}\right), \quad i \psi_{2}^{\prime \prime}=\frac{1}{\sqrt{2}}\left(\psi_{2}+\psi_{1}^{*}\right) .
\end{aligned}
$$

The four components of the Dirac spinors $\bar{\Psi}, \Psi$ are all independent variables. By convention, we can assume $\psi_{1}^{*}, \psi_{2}^{*}$ to be complex conjugated to $\psi_{1}, \psi_{2}$. In this case $\bar{\Psi}$ is the hermitian conjugated to $\Psi$. The advantage of the transformation from Majorana to Dirac formulation is that we gain in the latter case the gauge symmetry which can be useful in some cases [22]. The continuum-limit formulations like (6.10) and (7.2) captures all relevant features of the exact lattice theory at low momenta, or at large space scales, which is only important for the critical-point singularities in thermodynamic functions near $T_{c}$. For the simplest case of the standard rectangular lattice, the continuum limit was used to study the behaviour of the 2DIM spin correlation functions at large separation in [15,16]. Another important field of application of the continuum-limit formulation are the Ising models with quenched disorder [20-23].

In conclusion we note that the singular part of free energy and specific heat can be extracted, in the exact form, from (6.10) and/or (7.2). For instance, starting from (7.2), we find:

$$
\begin{aligned}
-\beta f_{\text {sing }} & =\frac{1}{2} \ln Q^{2}=\frac{1}{2} \int d^{2} x \ln Q^{2}(x)=\frac{1}{2} \int \frac{d^{2} p}{(2 \pi)^{2}} \ln Q^{2}(p) \\
& =\frac{1}{8 \pi^{2}} \int d^{2} p \ln \left(\bar{m}^{2}+p^{2}\right)=\frac{\pi}{8 \pi^{2}} \bar{m}^{2} \ln \frac{\text { const }}{\overline{\mathrm{m}}^{2}}+\ldots
\end{aligned}
$$

This is equivalent to equation (5.9) obtained directly from the exact expression for the lattice free energy (4.6), taking into account the definition of the effective mass given in (6.5).

\section{CONCLUSIONS}

We have discussed some aspects of a simple fermionic interpretation of the 2D Ising models in terms of the anticommuting integrals. For any planar 2D Ising model, the partition function can be expressed as a fermionic
Gaussian integral. The analytic solutions for regular lattices then easily follow by transformation to the momentum space. The continuum limit field-theoretical formulations for the 2D Ising models also can be deduced readily from the exact lattice fermionic integral for the partition function. The differences between particular lattices are merely adsorbed, in the field-theoretical limit, in the definition of the effective mass. Finally, the freefermion interpretation for the 2D Ising model shows that this model, which in its original formulation is a rather discrete combinatorial problem, can be placed, in fact, into a common range of the typical models of quantum statistics and solid state physics. 
[1] L. Onsager, Phys. Rev. 65, 117 (1944).

[2] K. Huang, Statistical Mechanics (Wiley, New York, 1963).

[3] A. Isihara, Statistical Physics (Academic Press, New York, 1971).

[4] R. P. Feynman, Statistical Mechanics (Benjamin, Mass., 1972).

[5] D. C. Mattis, The Theory of Magnetism II (Springer, Berlin, 1985).

[6] K. Wilson, Rev. Mod. Phys. 55, 583 (1983).

[7] T. D. Schultz, D. C. Mattis, E. H. Lieb, Rev. Mod. Phys. 36, 856 (1964).

[8] C. A. Hurst, H. S. Green, J. Chem. Phys. 33, 1059 (1960).

[9] F. A. Berezin, The Method of Second Quantization (Academic, New York, 1966).

[10] F. A. Berezin, Usp. Matem. Nauk 24, 3 (1969).

[11] E. Fradkin, M. Srednicki, L. Susskind, Phys. Rev. D 21, 2885 (1980).

[12] S. Samuel, J. Math. Phys. 21, 2806 (1980).

[13] C. Itzykson, Nucl. Phys. B 210 [FS6], 448 (1982).

[14] A. I. Bugrij, Preprints ITP-83-77P, Kiev, 1983; ITP85-114P, Kiev, 1985.

[15] J. B. Zuber, C. Itzykson, Phys. Rev. D 15, 2875 (1977).

[16] C. Itzykson, J. M. Drouffe, Statistical Field Theory (Cambridge Univ. Press, Cambridge, 1989).
[17] V. N. Plechko, Dokl. Akad. Nauk SSSR 281, 834 (1985) [Sov. Phys. - Doklady, 30, 271 (1985)].

[18] V. N. Plechko, Teor. Mat. Fiz. 64, 150 (1985). [Sov. Phys. Theor. Math. Phys. 64, 748 (1985)].

[19] V. N. Plechko, Physica A 152, 51 (1988).

[20] V. S. Dotsenko, V. S. Dotsenko, Sov. Phys. JETP Lett. 33, 37 (1981).

[21] V. S. Dotsenko, V. S. Dotsenko, Adv. Phys. 32129 (1983).

[22] B. N. Shalaev, Phys. Rep. 237, 129 (1994).

[23] G. Jug, B. N. Shalaev, Phys. Rev. B 54, 3442 (1996).

[24] R. Hayn, V. N. Plechko, J. Phys. A 27, 4753 (1994).

[25] V. N. Plechko, I. K. Sobolev, Phys. Lett. A 157, 335 (1991).

[26] V. N. Plechko, I. K. Sobolev, Physica A 197, 323 (1993).

[27] V. N. Plechko, In: Proceedings of the VII International Conference on Symmetry Methods in Physics, Dubna, July 10-16, 1995. Ed. by A. N. Sissakian and G. S. Pogosyan (JINR 96-224, Dubna, 1996), Vol. 2, p. 443-450. hep-th/9607053.

[28] V. N. Plechko, In: Proceedings of the $V$ International Conference on Path Integrals from meV to $M e V$, Dubna, May 27-31, 1996. Ed. by V. S. Yarunin and M. A. Smondyrev (JINR 96-321, Dubna, 1996), p. 295299. hep-th/9609044.

\title{
ФЕРМІОННІ ІНТЕГРАЛИ ТА АНАЛІТИЧНІ РОЗВ'ЯЗКИ ДЛЯ ДВОВИМІРНИХ МОДЕЛЕЙ ІЗІНГА
}

\author{
В. Н. Плєчко \\ Об’еднаний інститут ядерних дослідэсенъ, лабораторія теоретичної фізики ім. Боголюбова \\ Росіл, Московсъка область, 141980, Дубна
}

\begin{abstract}
Розглянуто деякі аспекти ферміонної інтерпретації двовимірної моделі Ізінга. Використано поняття інтеграла за антикомутуючими грассмановими змінними. Для простих та складніших двовимірних ізінгових граток статистичну суму можна виразити через ферміонний гаусів інтеграл. Таким самим чином двовимірну модель Ізінга можна переформулювати як теорію вільних ферміонів на гратці. Тоді для регулярних граток легко вивести аналітичний розв'язок переходом до імпульсного простору для ферміонів. Також наводяться ефективні теоретико-польові (в границі континууму) ферміонні формулювання для двовимірних моделей Ізінга біля критичної точки.
\end{abstract}

